\title{
GENERALIZED MAXIMUM PRINCIPLE IN OPTIMAL CONTROL
}

\author{
E. R. AVAKOV, G. G. MAGARIL-IL'YAEV
}

For an optimal control problem, the concept of a strong local infimum is introduce, for which necessary conditions consisting of some family of "maximum principles" are formulated. If a function delivers a strong local minimum in this problem (and therefore, a strong local infimum), then this family contains the classical Pontryagin maximum principle (see [1], [2]). As a corollary, we derive generalized necessary conditions for a strong local minimum for a problem of the calculus of variations. Examples are given to show that the necessary conditions obtained in the present paper generalize and strengthen classical results.

It is worth noting that some ideas for necessary conditions of this kind are contained in the book by R. V. Gamkrelidze [3], where the Pontryagin maximum principle is derived as a corollary to the maximum principle for a more general problem stated in terms of generalized controls. We also employ this idea, but from somewhat different positions and in a less general setting, when the generalized controls are sliding regime control. On the one hand, this constraint is quite sufficient for our purposes, and on the other hand, it leads to a simpler set of tools.

Let $\left[t_{0}, t_{1}\right]$ be a line interval, $U$ be a nonempty subset of $\mathbb{R}^{r}, \varphi: \mathbb{R} \times$ $\mathbb{R}^{n} \times \mathbb{R}^{r} \rightarrow \mathbb{R}^{n}$ be a mapping of variables $t \in \mathbb{R}, x \in \mathbb{R}^{n}$ and $u \in U$, and $f: \mathbb{R}^{n} \times \mathbb{R}^{n} \rightarrow \mathbb{R}^{m_{1}}, g: \mathbb{R}^{n} \times \mathbb{R}^{n} \rightarrow \mathbb{R}^{m_{2}}$ be mappings of variables $\zeta_{i} \in \mathbb{R}^{n}, i=1,2$.

Consider the following optimal control problem

$$
\begin{aligned}
f_{0}\left(x\left(t_{0}\right), x\left(t_{1}\right)\right) \rightarrow \min , \quad \dot{x}=\varphi(t, x, u(t)), & u(t) \in U, \\
f\left(x\left(t_{0}\right), x\left(t_{1}\right)\right) \leq 0, & g\left(x\left(t_{0}\right), x\left(t_{1}\right)\right)=0,
\end{aligned}
$$

where $x(\cdot) \in A C\left(\left[t_{0}, t_{1}\right], \mathbb{R}^{n}\right)$ (is an absolutely continuous vector function on $\left.\left[t_{0}, t_{1}\right]\right)$ and $u(\cdot) \in L_{\infty}\left(\left[t_{0}, t_{1}\right], \mathbb{R}^{r}\right)$.

In what follows we assume that the mapping $\varphi$ is continuous together with its derivative with respect to $x$ on $\mathbb{R} \times \mathbb{R}^{n} \times \mathbb{R}^{r}$, and the mappings $f$ and $g$ are continuously differentiable on $\mathbb{R}^{n} \times \mathbb{R}^{n}$. 
A function $x(\cdot) \in A C\left(\left[t_{0}, t_{1}\right], \mathbb{R}^{n}\right)$ is called admissible in problem (1) if $f\left(x\left(t_{0}\right), x\left(t_{1}\right)\right) \leq 0, g\left(x\left(t_{0}\right), x\left(t_{1}\right)\right)=0$ and there exists $u(\cdot) \in$ $L_{\infty}\left(\left[t_{0}, t_{1}\right], \mathbb{R}^{r}\right)$ such that $\dot{x}(t)=\varphi(t, x(t), u(t))$ and $u(t) \in U$ for almost all $t \in\left[t_{0}, t_{1}\right]$,

Definition. We say that a function $\widehat{x}(\cdot) \in C\left(\left[t_{0}, t_{1}\right], \mathbb{R}^{n}\right)$ delivers a strong local infimum in problem (1) if $f\left(\widehat{x}\left(t_{0}\right), \widehat{x}\left(t_{1}\right)\right) \leq 0$, $g\left(\widehat{x}\left(t_{0}\right), \widehat{x}\left(t_{1}\right)\right)=0$, there exist a neighbourhood $V$ of the point $\widehat{x}(\cdot)$ and a sequence $\left\{x_{N}(\cdot)\right\}, N \in \mathbb{N}$, of admissible functions in (1) such that $f_{0}\left(x\left(t_{0}\right), x\left(t_{1}\right)\right) \geq f_{0}\left(\widehat{x}\left(t_{0}\right), \widehat{x}\left(t_{1}\right)\right)$ for any admissible function $x(\cdot) \in V$ and $x_{N}(\cdot)$ converges uniformly to $\widehat{x}(\cdot)$ as $N \rightarrow \infty$.

Clearly, if a pair $(\widehat{x}(\cdot), \widehat{u}(\cdot))$ delivers a strong minimum in problem $(1)$, then $\widehat{x}(\cdot)$ is a strong local infimum in this problem. On the other hand, if a function $\widehat{x}(\cdot)$ delivers a strong local infimum in $(1), \widehat{x}(\cdot)$ is admissible, and $\widehat{u}(\cdot)$ is the corresponding control, then the pair $(\widehat{x}(\cdot), \widehat{u}(\cdot))$ delivers a strong minimum in this problem.

Given arbitrary $k \in \mathbb{N}$ and tuples $\bar{u}(\cdot)=\left(u_{1}(\cdot), \ldots, u_{k}(\cdot)\right) \in$ $\left.\left(L_{\infty}\left(\left[t_{0}, t_{1}\right]\right), \mathbb{R}^{r}\right)\right)^{k}$ and $\bar{\alpha}(\cdot)=\left(\alpha_{1}(\cdot), \ldots, \alpha_{k}(\cdot)\right) \in\left(L_{\infty}\left(\left[t_{0}, t_{1}\right]\right)\right)^{k}$, where $\alpha_{i}(t) \geq 0, \alpha_{i}(t) \neq 0, i=1, \ldots, k$, and $\sum_{i=1}^{k} \alpha_{i}(t)=1$ for almost all $t \in\left[t_{0}, t_{1}\right]$, we associate with the control system specifying the constraints in problem (1) the following extended (relaxation) control system

$$
\begin{aligned}
& \dot{x}=\sum_{i=1}^{k} \alpha_{i}(t) \varphi\left(t, x, u_{i}(t)\right), \quad u_{i}(t) \in U, \quad i=1, \ldots, k \\
& f\left(x\left(t_{0}\right), x\left(t_{1}\right)\right) \leq 0, \quad g\left(x\left(t_{0}\right), x\left(t_{1}\right)\right)=0 .
\end{aligned}
$$

A triple $(x(\cdot), \bar{u}(\cdot), \bar{\alpha}(\cdot))\left(x(\cdot) \in A C\left(\left[t_{0}, t_{1}\right], \mathbb{R}^{n}\right)\right)$ is called admissible for system (2) if it satisfies all constraints in (2).

Let us introduce some notation. We let $\langle\lambda, x\rangle=\sum_{i=i}^{n} \lambda_{i} x_{i}$ denote a linear functional $\lambda=\left(\lambda_{1}, \ldots, \lambda_{n}\right) \in\left(\mathbb{R}^{n}\right)^{*}$ evaluated at a point $x=$ $\left(x_{1}, \ldots, x_{n}\right)^{T} \in \mathbb{R}^{n}$ ( $T$ is the transpose). By $\left(\mathbb{R}^{n}\right)_{+}^{*}$ we denote the set of positive functionals on $\mathbb{R}^{n}$. The adjoint operator to a linear operator $\Lambda: \mathbb{R}^{n} \rightarrow \mathbb{R}^{m}$ is denoted by $\Lambda^{*}$.

Given a fixed function $\widehat{x}(\cdot)$, the partial derivatives of mappings $f$ and $g$ with respect to $\zeta_{1}$ and $\zeta_{2}$ at a point $\left(\widehat{x}\left(t_{0}\right), \widehat{x}\left(t_{1}\right)\right)$ will be briefly denoted by $\widehat{f}_{\zeta_{i}}$ and $\widehat{g}_{\zeta_{i}}, i=1,2$, respectively.

Theorem 1 (generalized maximum principle). If a function $\widehat{x}(\cdot) \in$ $A C\left(\left[t_{0}, t_{1}\right], \mathbb{R}^{n}\right)$ delivers a strong local infimum in problem $(1)$, then for any $k \in \mathbb{N}, \widehat{\bar{u}}(\cdot)=\left(\widehat{u}_{1}(\cdot), \ldots, \widehat{u}_{k}(\cdot)\right)$ and $\widehat{\bar{\alpha}}(\cdot)=\left(\widehat{\alpha}_{1}(\cdot), \ldots, \widehat{\alpha}_{k}(\cdot)\right)$ such that the triple $(\widehat{x}(\cdot), \widehat{\bar{u}}(\cdot), \widehat{\bar{\alpha}}(\cdot))$ is admissible for the control 
system (2), there exist a nonzero tuple $\left(\lambda_{0}, \lambda_{f}, \lambda_{g}\right) \in \mathbb{R}_{+} \times\left(\mathbb{R}^{m_{1}}\right)_{+}^{*} \times$ $\left(\mathbb{R}^{m_{2}}\right)^{*}$ and a vector function $p(\cdot) \in A C\left(\left[t_{0}, t_{1}\right],\left(\mathbb{R}^{n}\right)^{*}\right)$ such that the following conditions hold:

1) the stationarity condition with respect to $x(\cdot)$

$$
\dot{p}(t)=-p(t) \sum_{i=1}^{k} \widehat{\alpha}_{i}(t) \varphi_{x}\left(t, \widehat{x}(t), \widehat{u}_{i}(t)\right),
$$

2) the transversality condition

$$
p\left(t_{0}\right)=\lambda_{0} \widehat{f}_{0 \zeta_{1}}+\widehat{f}_{\zeta_{1}}^{*} \lambda_{f}+\widehat{g}_{\zeta_{1}}^{*} \lambda_{g}, \quad p\left(t_{1}\right)=-\lambda_{0} \widehat{f}_{0 \zeta_{2}}-\widehat{f}_{\zeta_{2}}^{*} \lambda_{f}-\widehat{g}_{\zeta_{2}}^{*} \lambda_{g}
$$

3) the complementary slackness condition

$$
\left\langle\lambda_{f}, f\left(\widehat{x}\left(t_{0}\right), \widehat{x}\left(t_{1}\right)\right)\right\rangle=0,
$$

4) the maximum condition for almost all $t \in\left[t_{0}, t_{1}\right]$

$$
\begin{gathered}
\max _{u \in U} \widehat{\alpha}_{i}(t)\langle p(t), \varphi(t, \widehat{x}(t), u)\rangle=\widehat{\alpha}_{i}(t)\left\langle p(t), \varphi\left(t, \widehat{x}(t), \widehat{u}_{i}(t)\right)\right\rangle, \\
\qquad i=1, \ldots, k, \\
\max _{u \in U}\langle p(t), \varphi(t, \widehat{x}(t), u)\rangle=\langle p(t), \dot{\widehat{x}}(t)\rangle .
\end{gathered}
$$

Moreover, if for some $k \in \mathbb{N}$ and a triple $(\widehat{x}(\cdot), \bar{u}(\cdot), \bar{\alpha}(\cdot))$ admissible for the control system (2), conditions 1)-4) hold only when $\lambda_{0} \neq 0$, then there exists a sequence of functions $x_{N}(\cdot), N \in \mathbb{N}$ admissible in problem (1) such that $x_{N}(\cdot) \rightarrow \widehat{x}(\cdot)$ as $N \rightarrow \infty$ uniformly on $\left[t_{0}, t_{1}\right]$.

The first assertion of the theorem constitutes a family of relations (parameterized by all possible finite tuples $(\widehat{\bar{u}}(\cdot), \widehat{\bar{\alpha}}(\cdot))$ such that the triple $(\widehat{x}(\cdot), \widehat{\bar{u}}(\cdot), \widehat{\bar{\alpha}}(\cdot))$ is admissible for the control system $(2))$, of which each has the form of a maximum principle. Furthermore, if $(\widehat{x}(\cdot), \widehat{u}(\cdot))$ is a strong minimum in problem (1), then this family contains (with $k=1, \widehat{u}_{1}(\cdot)=\widehat{u}(\cdot)$ and $\widehat{\alpha}_{1}(\cdot)=1$ ) the classical Pontryagin maximum principle.

As a corollary to Theorem 1 we obtain generalized conditions for strong local minimum in the simplest problem of the classical calculus of variations.

Let a function $L: \mathbb{R} \times \mathbb{R}^{n} \times \mathbb{R}^{n} \rightarrow \mathbb{R}$ of variables $t \in \mathbb{R}, x \in \mathbb{R}^{n}$ and $\dot{x} \in \mathbb{R}^{n}$ be continuous together with its partial derivatives with respect to $x, \dot{x}$, and $x_{i} \in \mathbb{R}^{n}, i=0,1$. Consider the problem

$$
\int_{t_{0}}^{t_{1}} L(t, x(t), \dot{x}(t)) d t \rightarrow \min , \quad x\left(t_{0}\right)=x_{0}, \quad x\left(t_{1}\right)=x_{1} .
$$


Given a fixed $\widehat{x}(\cdot)$, we write for brevity $\widehat{L}(t)=L(t, \widehat{x}(t), \dot{\widehat{x}}(t))$, and similarly for the derivatives of $L$ with respect to $x$ and $\dot{x}$.

We let $\mathcal{A}^{k}$ denote the set of tuples $\bar{\alpha}(\cdot)=\left(\alpha_{1}(\cdot), \ldots, \alpha_{k}(\cdot)\right)$ introduced before the definition of the control system (2).

Theorem 2. If a function $\widehat{x}(\cdot) \in A C\left(\left[t_{0}, t_{1}\right], \mathbb{R}^{n}\right)$ delivers a strong local minimum in problem $(3)$, then $\widehat{L}_{\dot{x}}(\cdot) \in A C\left(\left[t_{0}, t_{1}\right], \mathbb{R}^{n}\right)$ and for any $k \in$ $\mathbb{N},\left(\widehat{u}_{1}(\cdot), \ldots, \widehat{u}_{k}(\cdot)\right) \in\left(L_{\infty}\left(\left[t_{0}, t_{1}\right], \mathbb{R}^{n}\right)\right)^{k}$ and $\left(\widehat{\alpha}_{1}(\cdot), \ldots, \widehat{\alpha}_{k}(\cdot)\right) \in \mathcal{A}^{k}$ such that, for almost all $t \in\left[t_{0}, t_{1}\right]$

$$
\dot{\widehat{x}}(t)=\sum_{i=1}^{k} \widehat{\alpha}_{i}(t) \widehat{u}_{i}(t) \text { and } \widehat{L}(t)=\sum_{i=1}^{k} \widehat{\alpha}_{i}(t) L\left(t, \widehat{x}(t), \widehat{u}_{i}(t)\right) \text {, }
$$

the following conditions are satisfied:

1) the generalized Euler equation

$$
-\frac{d}{d t} \widehat{L}_{\dot{x}}(t)+\sum_{i=1}^{k} \widehat{\alpha}_{i}(t) L_{x}\left(t, \widehat{x}(t), \widehat{u}_{i}(t)\right)=0,
$$

2) the generalized Weierstrass condition

$$
\begin{array}{r}
L(t, \widehat{x}(t), u)-L\left(t, \widehat{x}(t), \widehat{u}_{i}(t)\right)-\left\langle L_{\dot{x}}\left(t, \widehat{x}(t), \widehat{u}_{i}(t)\right), u-\widehat{u}_{i}(t)\right\rangle \geq 0, \\
i=1, \ldots, k,
\end{array}
$$

for all $u \in \mathbb{R}^{n}$.

3) If $L$ is twice differentiable with respect to $\dot{x}$, then the generalized Legendre condition holds

$$
\widehat{L}_{\dot{x} \dot{x}}\left(t, \widehat{x}(t), \widehat{u}_{i}(t)\right) \geq 0, \quad i=1, \ldots, k .
$$

It is clear that for $k=1, \widehat{u}_{1}(\cdot)=\dot{\widehat{x}}(\cdot)$ and $\widehat{\alpha}_{1}(\cdot)=1$ conditions (4) hold trivially and conditions 1$)-3$ ) pass into the classical strong minimum conditions in problem (3).

The first example illustrates how a strong local infimum can be found with the help of Theorem 1.

Example 1. Let $f:[0,1] \rightarrow \mathbb{R}, g: \mathbb{R} \rightarrow \mathbb{R}, m$ be an even number. Consider the optimal control problem

$$
\begin{array}{r}
J(x(\cdot), u(\cdot))=\int_{0}^{1}\left((x(t)-f(t))^{m}+g(u(t))\right) d t \rightarrow \min , \quad \dot{x}=u, \\
|u(t)| \geq 1, \quad x(0)=0, \quad x(1)=f(1) .
\end{array}
$$


We shall assume that the function $f$ is absolutely continuous, $f(0)=$ 0 , and $|\dot{f}(t)| \leq 1,|\dot{f}(t)| \neq 1$ for almost all $t \in[0,1]$. We also assume that $g$ is continuous on $\mathbb{R}, g(-1)=g(1)$, and $g(u)>g(1)$ for $|u|>1$.

Our aim is to evaluate the infimum of the functional $J(x(\cdot), u(\cdot)$ ) and find a sequence of admissible pairs $\left(x_{N}(\cdot), u_{N}(\cdot)\right), N \in \mathbb{N}$ in problem (5) on which the sequence $J\left(x_{N}(\cdot), u_{N}(\cdot)\right)$ converges to this infimum.

We transform problem (5) to the equivalent Mayer problem

$$
\begin{aligned}
x_{2}(1)-x_{2}(0) \rightarrow \min , \quad \dot{x}_{1}=u, \quad \dot{x}_{2}=\left(x_{1}-f(t)\right)^{m}+g(u), \\
\\
|u(t)| \geq 1, \quad x_{1}(0)=0, \quad x_{1}(1)=f(1) .
\end{aligned}
$$

Using Theorem 1, we shall try to find a function which delivers a strong local infimum in this problem. If such a function $\widehat{\bar{x}}(\cdot)=$ $\left(\widehat{x}_{1}(\cdot), \widehat{x}_{2}(\cdot)\right)$ is found, then by definition $x_{2}(1)-x_{2}(0) \geq \widehat{x}_{2}(1)-\widehat{x}_{2}(0)$ for all admissible functions $\bar{x}(\cdot)$ from some neighbourhood of $\widehat{\bar{x}}(\cdot)$ and there exists a sequence of admissible for (6) functions $\bar{x}_{N}(\cdot)=$ $\left(x_{1 N}(\cdot), x_{2 N}(\cdot)\right)$ that converges uniformly to $\widehat{\bar{x}}(\cdot)$ as $N \rightarrow \infty$. It follows that $J(x(\cdot), u(\cdot)) \geq \widehat{x}_{2}(1)-\widehat{x}_{2}(0)$ for all admissible pairs in problem $(5)$ in which $x(\cdot)$ lies in some neighbourhood of $\widehat{x}_{1}(\cdot)$. Setting $x_{1 N}(\cdot)=x_{N}(\cdot)$, we find a sequence of pairs $\left(x_{N}(\cdot), u_{N}(\cdot)\right)$ admissible in problem (5), where $u_{N}(\cdot)=\dot{x}_{N}(\cdot)$, such that $J\left(x_{N}(\cdot), u_{N}(\cdot)\right) \rightarrow$ $\widehat{x}_{2}(1)-\widehat{x}_{2}(0)$ as $N \rightarrow \infty$.

We apply Theorem 1 with $k=2$. By this theorem if tuples $\left(\widehat{\alpha}_{1}(\cdot), \widehat{\alpha}_{2}(\cdot)\right)$ and $\left(\widehat{u}_{1}(\cdot), \widehat{u}_{2}(\cdot)\right)$ are such that

$$
\begin{aligned}
& \dot{\widehat{x}}_{1}(t)=\widehat{\alpha}_{1}(t) \widehat{u}_{1}(t)+\widehat{\alpha}_{2}(t) \widehat{u}_{2}(t), \\
& \dot{\widehat{x}}_{2}(t)=\widehat{\alpha}_{1}(t) g\left(\widehat{u}_{1}(t)\right)+\widehat{\alpha}_{2}(t) g\left(\widehat{u}_{2}(t)\right)+\left(\widehat{x}_{1}(t)-f(t)\right)^{m}
\end{aligned}
$$

and $\widehat{x}_{1}(0)=0, \widehat{x}_{1}(1)=f(1)$, then there exist a nonzero set of Lagrange multipliers $\left(\lambda_{0}, \lambda_{1}, \lambda_{2}\right)$, where $\lambda_{0} \geq 0$, and an absolutely continuous function $p(\cdot)$, such that

$$
\begin{array}{r}
\dot{p}_{1}=-p_{2} m(\widehat{x}(t)-f(t))^{m-1}, \quad \dot{p}_{2}=0, \quad p_{1}(0)=\lambda_{1}, \quad p_{1}(1)=-\lambda_{2} \\
p_{2}(0)=p_{2}(1)=-\lambda_{0}
\end{array}
$$

and

$$
\max _{u \in U}\left(p_{1}(t) u+p_{2}(t)\left((\widehat{x}(t)-f(t))^{m}+g(u)\right)\right)=p_{1}(t) \dot{\widehat{x}}_{1}(t)+p_{2}(t) \dot{\widehat{x}}_{2}(t)
$$

for almost all $t \in\left[t_{0}, t_{1}\right]$.

By examining relations (7), (8) and (9) one can show that they define uniquely, up to an additive constant, the function $\left(\widehat{x}_{1}(\cdot), \widehat{x}_{2}(\cdot)\right)$ (where $\widehat{x}_{1}(t)=f(t), \widehat{x}_{2}(t)=g(1) t+c$ for any $c \in \mathbb{R}$ and all $\left.t \in\left[t_{0}, t_{1}\right]\right)$ and 
(assuming $\left.\lambda_{0}=1\right)$ the Lagrange multipliers $\lambda=(1,0,0)$ and $p=(0,1)$. Here it suffices to put $\widehat{u}_{1}(t) \equiv 1, \widehat{u}_{2}(t) \equiv-1$, which gives $\widehat{\alpha}_{1}(t)=$ $(1+\dot{f}(t)) / 2, \widehat{\alpha}_{2}(t)=(1-\dot{f}(t)) / 2$ for almost all $t \in\left[t_{0}, t_{1}\right]$.

So, $t \mapsto(f(t), g(1) t+c)$ is the only trajectory suspected for a strong local infimum in problem (6) for each $c \in \mathbb{R}$. Note that this trajectory is not admissible for this problem. We claim that it delivers a strong local infimum.

Indeed, $\lambda_{0} \neq 0$, for otherwise relations (8) and (9) would hold only for $\lambda_{1}=\lambda_{2}=0$. Hence, by the second assertion of the theorem there exists a sequence of functions $\left(x_{1 N}(\cdot), x_{2 N}(\cdot)\right)$ which are admissible for problem (6) and uniformly converge to $\left(\widehat{x}_{1}(\cdot), \widehat{x}_{2}(\cdot)\right)$ as $N \rightarrow \infty$.

Additionally, it is clear that $x_{2}(1)-x_{1}(0) \geq g(1)=\widehat{x}_{2}(1)-\widehat{x}_{2}(0)$ for any admissible function $x(\cdot)=\left(x_{1}(\cdot), x_{2}(\cdot)\right)$, and hence by definition $\widehat{x}(\cdot)$ delivers the global infimum in problem (6). Hence, the infimum of the functional $J(x(\cdot), u(\cdot))$ is $g(1)$, and by the above there exists a sequence of admissible pairs in problem (5) on which this functional converges to $g(1)$.

Let us construct directly a sequence of admissible pairs $\left(x_{N}(\cdot) u_{N}(\cdot)\right)$ in problem (5) such that $J\left(x_{N}(\cdot), u_{N}(\cdot)\right) \rightarrow g(1)$ as $N \rightarrow \infty$. Let $N \in \mathbb{N}$. We split the interval $[0,1]$ into $N$ intervals: $[s / N,(s+1) / N], s=0, \ldots, N-1$. Define $b_{N}(s)=f(s / N)-(s / N)$ and $c_{N}(s)=f((s+1) / N)+(s+1) / N, s=0, \ldots, N-1$. It is easily checked that $\left(\left(c_{N}(s)-b_{N}(s)\right) / 2\right) \in[s / N,(s+1) / N], s=0, \ldots, N-1$.

Consider the sequence $x_{N}(\cdot)$ defined by

$$
x_{N}(t)= \begin{cases}t+b_{N}(s), & t \in\left[s / N,\left(c_{N}(s)-b_{N}(s)\right) / 2\right], \\ -t+c_{N}(s), & t \in\left[\left(c_{N}(s)-b_{N}(s)\right) / 2,(s+1) / N\right]\end{cases}
$$

$s=0, \ldots, N-1$. Each $x_{N}(t)$ is a broken line (with slopes \pm 1 of the segments and which interpolates $f(\cdot)$ at the points $s / N$, $s=0, \ldots, N)$ and $x_{N}(t)$ uniformly converges to $f(\cdot)$. The sequence of pairs $\left(x_{N}(\cdot), u_{N}(\cdot)\right)$, where $u_{N}(\cdot)=\dot{x}_{N}(\cdot)$, is admissible in problem (5), because $\left|u_{N}(t)\right|=1$ for almost all $t \in\left[t_{0}, t_{1}\right]$, and since $J\left(x_{N}(\cdot), u_{N}(\cdot)\right) \rightarrow g(1)$ as $N \rightarrow \infty$, which is clear.

The following example shows that even in the classical setting the above assertions are capable of delivering additional information about the strong minimum compared to with known necessary conditions. 
Example 2. Let $L: \mathbb{R} \times \mathbb{R} \rightarrow \mathbb{R}$. Consider the following variational calculus problem

$$
\int_{0}^{1} L(x, \dot{x}) d t \rightarrow \min , \quad x(0)=x(1)=0 .
$$

Assume that the function $L$ is continuously differentiable, $L_{x}(0,0)=$ $L_{\dot{x}}(0,0)=0$ and $L(0, \dot{x})=0$ for any $\dot{x}$ (a typical situation when $L(x, \dot{x})=x f(\dot{x})$, where the function $f$ is continuously differentiable and $f(0)=0)$.

It is an elementary matter to verify that the function $\widehat{x}(\cdot)=0$ satisfies the Pontryagin maximum principle $(\dot{x}=u, u \in U=\mathbb{R})$. The next result is proved using Theorem 2.

Proposition. If a function $\widehat{x}(\cdot)=0$ delivers a strong local minimum in problem (10), then the function $\dot{x} \rightarrow L_{x}(0, \dot{x})$ is linear.

Доказательство. We apply the theorem with $k=2$. It is clear that conditions (4) are satisfied for any $u_{1}<0, u_{2}>0$ and $\alpha_{1}=u_{2} /\left(u_{2}-u_{1}\right)$, $\alpha_{2}=-u_{1} /\left(u_{2}-u_{1}\right)$. Hence, the Euler equation holds, which in this case reads as

$$
u_{2} L_{x}\left(0, u_{1}\right)=u_{1} L_{x}\left(0, u_{2}\right) .
$$

Setting here $u_{1}=-1, u_{2}=1$, we find

$$
L_{x}(0,-1)=-L_{x}(0,1) .
$$

Let $u \in \mathbb{R}$ and $u \neq 0$. If $u<0$, then from (11) for $u_{1}=u$ and $u_{2}=1$ we find that

$$
L_{x}(0, u)=L_{x}(0,1) u \text {. }
$$

If $u>0$, then again from (11) with $u_{2}=u$ and $u_{1}=-1$ and taking into account (12), we have

$$
L_{x}(0, u)=-L_{x}(0,-1) u=L_{x}(0,1) u .
$$

If $u=0$, then by the condition $L_{x}(0,0)=0$, and so $L_{x}(0, u)=L_{x}(0,1) u$ for any $u \in \mathbb{R}$.

In fact a more general fact holds. Assume that in problem (10) $\dot{x}=u$ and $u(t) \in U$ for almost all $t \in\left[t_{0}, t_{1}\right]$, where $U$ is an arbitrary set, but $0 \in \operatorname{int} U$. No differentiability of $L$ with respect to $u$ is required. If $\widehat{x}(\cdot)=0$ delivers a strong local infimum in this problem, then using Theorem 1 and arguing as in the proposition, we find that the function $u \mapsto L_{x}(0, u)$ is linear on some interval with centre at the origin.

Thus, Theorem 1 is a strengthening of the Pontryagin maximum principle.

Acknowledgement. The authors are sincerely grateful to Revaz Valer'yanovich Gamkrelidze for helpful discussions. 


\section{СПИСОК ЛИТЕРАТУРЫ}

[1] L. S. Pontryagin; V. G. Boltyanskii, R. V. Gamkrelidze, and E. F. Mishchenko, The mathematical theory of optimal processes, Wiley, New York-London 1962, viii +360 pp.

[2] A. D. Ioffe and V. M. Tihomirov (Tikhomirov), Theory of extremal problems, Nauka, Moscow 1974; English transl., Stud. Math. Appl., vol. 6, North-Holland, Amsterdam-New York 1979.

[3] R. V. Gamkrelidze, Principles of optimal control theory, Tbilisi University Publishing House, Tbilisi 1977; English transl., Plenum Press, New YorkLondon 1978.

Institute of Control Sciences of the Russian Academy of Sciences

Moscow State University 